\title{
Methodological Issues Related to Thickness-Based Muscle Size Evaluation
}

\author{
Ryota Akagi ${ }^{1)}$, Soichiro Iwanuma ${ }^{2), 3)}$, Masato Fukuoka ${ }^{4)}$, Hiroaki Kanehisa ${ }^{5)}$, \\ Tetsuo Fukunaga ${ }^{5)}$ and Yasuo Kawakami ${ }^{6)}$ \\ 1) Department of Sports Sciences, Japan Institute of Sports Sciences \\ 2) Research Fellow of the Japan Society for the Promotion of Science \\ 3) Graduate School of Sport Sciences, Waseda University \\ 4) School of Sport Sciences, Waseda University \\ 5) National Institute of Fitness and Sports in Kanoya \\ 6) Faculty of Sport Sciences, Waseda University
}

\begin{abstract}
The purpose of this study was to highlight the issues related to thickness-based muscle size evaluation that is commonly done in field studies. The cross-sectional area, thickness (the vertical distance from the upper end of the elbow flexors to that of the humerus) and width (the horizontal distance from the left to the right end of the elbow flexors) of the elbow flexors at levels from the reference site $(60 \%$ of the upper arm length from the acromial process of the scapula to the lateral epicondyle of the humerus) to $5 \mathrm{~cm}$ distal to it were determined in 11 young men using magnetic resonance imaging, both at rest and during isometric elbow flexion at $30 \%$ of maximal voluntary contraction. During 30\% of maximal voluntary contraction, the thickness increased but the width decreased at each measurement site compared with those at rest. This was possibly due to difference in muscle slackness between both conditions. The correlation coefficients between the thickness and cross-sectional area for the elbow flexors were significantly lower at rest $(r=0.551-0.856)$ than during $30 \%$ of maximal voluntary contraction $(r=0.711-0.922)$. The present findings indicate that the thickness-based muscle size measurement at rest includes errors owing to the slackness of the resting muscles. J Physiol Anthropol 30(4): 169-174, 2011 http://www.jstage.jst.go.jp/browse/jpa2
\end{abstract}

[DOI: 10.2114/jpa2.30.169]

Keywords: muscle cross-sectional area, muscle width, isometric contraction, muscle slackness, magnetic resonance imaging.

\section{Introduction}

It is well known that muscle thickness is closely related to muscle cross-sectional area (CSA) (Abe et al., 1997; Sipila and Suominen, 1991, 1993) and volume (Miyatani et al., 2004), which are major determinants of muscle strength (Bamman et al., 2000; Gadeberg et al., 1999; Ikai and Fukunaga, 1968). Therefore, ultrasonic measurement of muscle thickness is used to evaluate muscle size (Akagi et al., 2010; Candow and Chilibeck, 2005; Ohata et al., 2008; Pillen et al., 2003, 2007 etc.) as an alternative to magnetic resonance imaging (MRI) and computed tomography which are not easily accessible for practical use in field studies dealing with large numbers of subjects. However, previous studies measured muscle thickness at rest. The cross-sectional shape of the relaxed muscle at rest sags due to slackness (Akagi et al., 2008). This phenomenon questions the accuracy of thickness-based muscle size measurement at rest, because muscle thickness is the distance within the muscle cross-sectional shape.

Muscle contraction is considered as a means to eliminate the slackness in a muscle. In parallel-fibered muscles such as the elbow flexors, marked increases in the thickness and CSA induced by contraction are shown (Akagi et al., 2008, 2009; Hodges et al., 2003). Additionally, by the use of MRI, Pappas et al. (2002) found a remarkable change in fascicle length of the biceps brachii muscle by low-intensity contractions of elbow flexion. These suggest that the slackness of the elbow flexors is taken away by the contractions, thereby changing the muscle thickness and CSA. Based on this, we quantified the cross-sectional shapes of the elbow flexors both at rest and during a low-intensity contraction by MRI and examined the thickness-CSA relationships in the two conditions. The main purpose of this study was to highlight the methodological issues relevant to thickness-based muscle size measurement.

\section{Methods}

\section{Subjects}

Eleven young males (age: $22.8( \pm 2.0) \mathrm{yr}$, body height: 172.2 $( \pm 5.1) \mathrm{cm}$, body mass: $62.8( \pm 5.9) \mathrm{kg}$; means ( \pm standard deviations, SDs)) voluntarily participated after being informed of all the risks associated with this study, which was approved 
by the Ethical Committee of the Faculty of Sport Sciences of Waseda University. The subjects were sedentary-or-moderately to highly active individuals but none was currently engaged in any type of regular exercise program. Written informed consent was obtained from each subject. They had no orthopedic abnormality in their right arms.

\section{Procedures}

The experimental setup is shown in Fig. 1. Each subject was instructed to lie in a supine position on the bed of an MRI system (Signa 1.5T, GE Medical Systems, USA) and the right arm was secured to a custom-made torque meter (VINE, Japan) using a non-elastic belt, with the elbow at $80^{\circ}$ (full extension $=0^{\circ}$ ) and the wrist fixed to the torque meter in a position halfway between supination and pronation. The size of the MRI gantry used in this study restricted the position of the subjects. It has been reported that the elbow flexor thickness and CSA increase with contraction when the elbow is flexed at $90^{\circ}$ (Akagi et al., 2008, 2009; Hodges et al., 2003), and that the elbow flexor strength is highest at $80^{\circ}-90^{\circ}$ (Hansen et al., 2003; Leedham and Dowling, 1995). To avoid the possibility of the right hand touching the MRI gantry when the elbow joint is flexed at $90^{\circ}$, therefore, the elbow joint angle of $80^{\circ}$ flexion was adopted as the test position. The torque meter was made of acetal copolymer, vinyl chloride, acrylic and polyamide, all of which were nonmagnetic. A custom-made optical fiber (Shinko Electric Wire, Japan) was attached to the lever arm of the torque meter to record strains around the lever

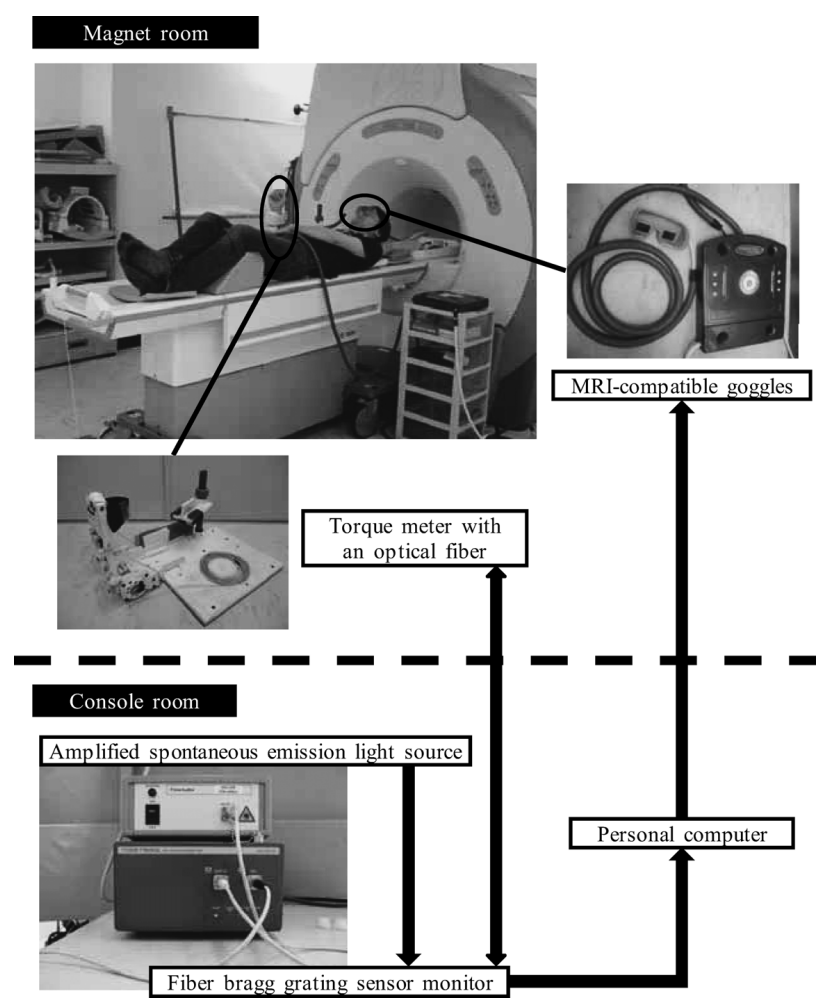

Fig. 1 Experimental setup. arm with a fiber bragg grating sensor monitor (FB200, Yokogawa Electric, Japan), combined with an amplified spontaneous emission light source (ASE-1550-25, FiberLabs, Japan). The strain data were sent to a personal computer (VGN-SZ80PS, Sony, Japan) at $100 \mathrm{~Hz}$ for calculation of torque. There was a strong linear relationship between torque and wavelength of the optical fiber $\left(R^{2}=0.99\right)$.

The subjects performed isometric maximal voluntary contraction (MVC) of elbow flexion for 3 seconds. The torque measurements were repeated two or three times with at least a 2-minute interval, and the highest value was adopted to calculate a target level. Afterwards, a series of cross-sectional images of the right arm was obtained using MRI with a 5-inch round surface coil attached to the side of the torque meter. This coil was not in contact with the right arm; thus, it did not influence the cross-sectional shape of elbow flexors. Transverse scans were performed with a conventional proton density weighted Fast Recovery Fast Spin echo technique (repetition time: $1300 \mathrm{~ms}$, echo time: $20 \mathrm{~ms}$, echo train length: 8, bandwidth: $31.25 \mathrm{kHz}$, number of excitations: 0.5 , slice thickness: $10 \mathrm{~mm}$, interspaced distance: $0 \mathrm{~mm}$, field of view: $160 \times 160 \mathrm{~mm}$ with $256 \times 160$ matrix). A marker was attached to the subjects' skin surface at $60 \%$ of the upper arm length from the acromial process of the scapula to the lateral epicondyle of the humerus (Abe et al., 1994), and this site was defined as the reference site. Within the device, the subjects maintained the aforementioned posture and wore MRIcompatible goggles (MRVision 2000, Resonance Technology, USA, with VisuaStim Digital's Controller, Resonance Technology, USA) that displayed a screen of the computer of the torque meter system (Fig. 1), in order to provide the subjects with visual feedback on their contraction level. Firstly, the series of cross-sectional images of the right arm were scanned at rest. Next, the same scan was made while the subjects sustained $30 \%$ of MVC of elbow flexion (30\%MVC) for about 20 seconds. During the scanning process, there was little variation in the contraction level $(30 \% \mathrm{MVC})$ for each subject. In each condition, the scan time was 18 seconds.

In all scanned images, the outlines of the elbow flexors (biceps brachii, brachialis, and brachioradialis) were digitized. The successive CSA and thickness (the vertical distance from the upper end of the elbow flexors to that of the humerus) values at levels from the reference site to $5 \mathrm{~cm}$ distal to it (every $1 \mathrm{~cm}$; 6 measurement sites) were manually quantified using image analysis software (Osiris 4.19, University Hospital of Geneva, Switzerland). The measurement sites were chosen so that the largest CSA was included, and the elbow flexor thickness was often measured within this range previously (Akagi et al., 2010; Arts et al., 2010; Reimers et al., 1998; Wakahara et al., 2010 etc.). In addition to the CSA and thickness, the width (the horizontal distance from the left to the right end of the elbow flexors) was also manually analyzed using the image analysis software as a parameter relevant to the cross-sectional shape (Fig. 2). Each measurement was carried out one time by an experienced tester (Akagi et al., 


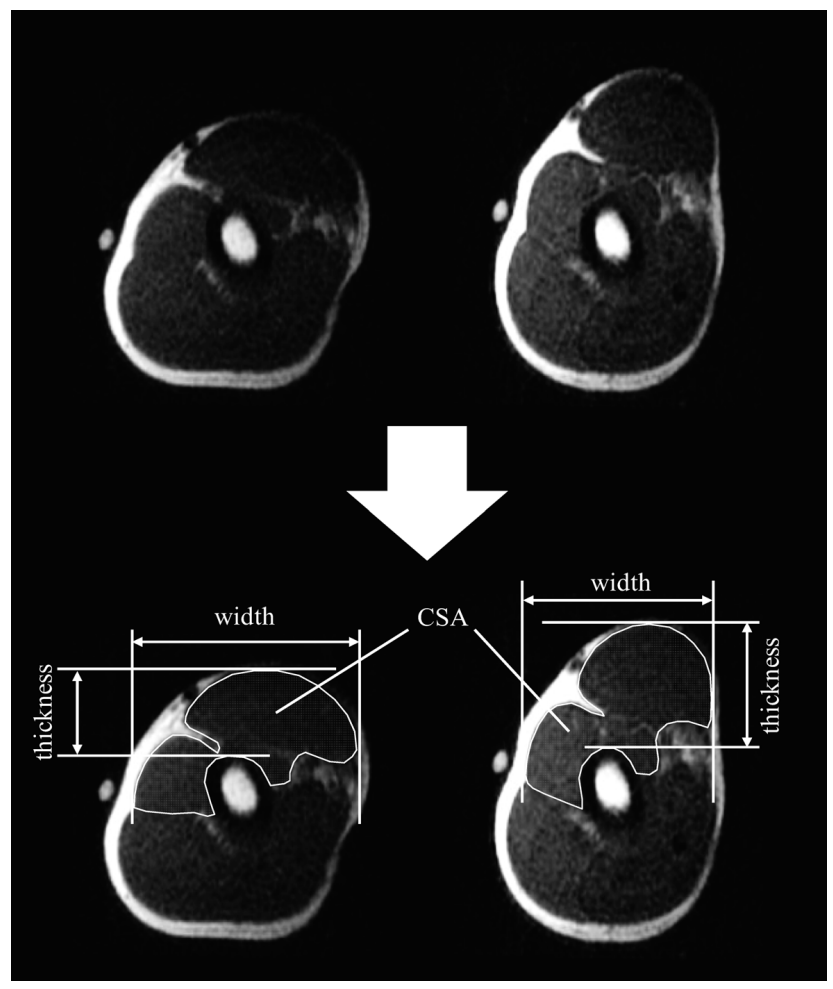

Fig. 2 MRI images of cross-sections of elbow flexors at rest (left) and during 30\%MVC (right).

MVC, maximal voluntary contraction.

2008, 2010). To verify the accuracy of analysis, the measurements of each variable at the reference site were carried out twice. The coefficient of variances $(\mathrm{CVs})$ of the two values for each variable were $<1.4 \%$ with intraclass correlation coefficients of $>0.986$.

In order to confirm the day-to-day reproducibility of the measurements, the above procedures were repeated for 3 subjects on another day. The CVs of the two measured values ( 3 men $\times 6$ measurement sites) were $<2.2 \%$ and the test-retest intraclass correlation coefficients were $>0.916$.

\section{Statistical analyses}

Descriptive data are presented as means \pm SDs. A two-way analysis of variance (ANOVA) ( 2 contraction levels $\times 6$ measurement sites) with repeated measures was used to test the effects of the contraction levels on all variables. When the interaction between the two factors was significant, a Student's paired $t$-test was conducted in each measurement site to test the differences between at rest and during 30\%MVC. At each measurement site, Pearson's product-moment correlation coefficients between the thickness and CSA both at rest and during $30 \% \mathrm{MVC}$ were calculated. The difference in the correlation coefficients between at rest and during $30 \% \mathrm{MVC}$ was analyzed with the Wilcoxon matched-pairs signed-ranks test. Statistical significance was set at $p<0.05$.
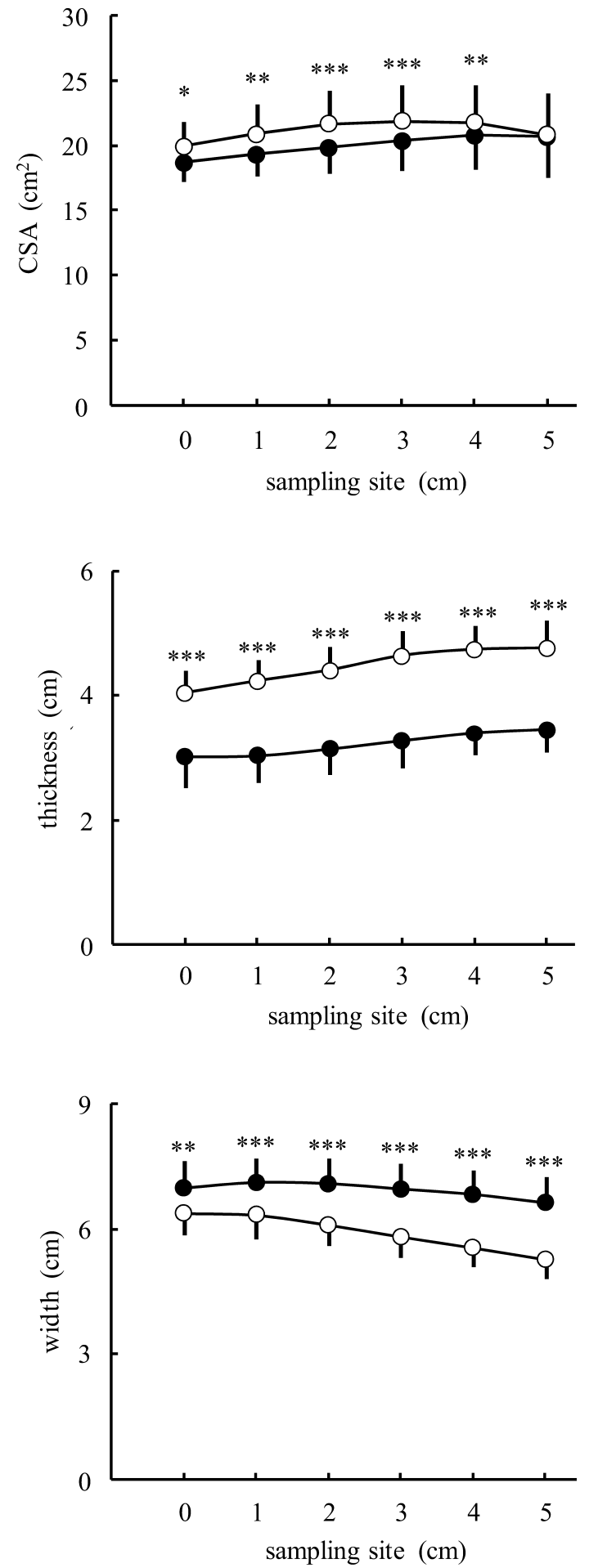

Fig. 3 Mean values of CSA, thickness and width of the elbow flexors at rest $(\bigcirc)$ and during 30\%MVC $(O)(n=11)$.

CSA, cross-sectional area; MVC, maximal voluntary contraction. The error bars correspond to standard deviations of the parameters. The abscissa shows the distance from the reference site $(60 \%$ of the upper arm length from the acromial process of the scapula to the lateral epicondyle of the humerus) in the distal direction. * $(p<0.05)$, ** $(p<0.01), * * *(p<0.001)$. Significant difference between at rest and during $30 \% \mathrm{MVC}$ at the same measurement site. 
Table 1 Correlation coefficients between thickness and CSA of the elbow flexors at rest and during $30 \% \mathrm{MVC}(n=11)$

\begin{tabular}{cll}
\hline Sampling site cm & Rest & $30 \% \mathrm{MVC}^{\dagger}$ \\
\hline 0 & 0.551 & $0.777^{* *}$ \\
1 & $0.697^{*}$ & $0.711^{*}$ \\
2 & $0.627^{*}$ & $0.781^{* *}$ \\
3 & $0.756^{* *}$ & $0.819^{* *}$ \\
4 & $0.817^{* *}$ & $0.922^{* *}$ \\
5 & $0.856^{* *}$ & $0.877^{* * *}$ \\
\hline
\end{tabular}

CSA, cross-sectional area; MVC, maximal voluntary contraction. The sampling site denotes distance from the reference site ( $60 \%$ of the upper arm length from the acromial process of the scapula to the lateral epicondyle of the humerus) in the distal direction. ${ }^{*}(p<0.05), * *(p<$ $0.01), * * *(p<0.001)$. Significant correlation between thickness and CSA. ${ }^{\dagger}(p<0.05)$ Significant difference from at rest.

\section{Results}

Figure 3 shows the CSA, thickness, and width of the elbow flexors at each measurement site. There were significant interactions between the contraction levels and measurement sites for all variables. The CSAs during 30\%MVC were significantly greater than those at rest except for the site $5 \mathrm{~cm}$ distal to the reference site. At all measurement sites, the thickness increased whereas the width decreased significantly.

The correlation coefficients between the thickness and the CSA at each measurement site are shown in Table 1. In the resting condition, there were significant correlations between the thickness and the CSA $(r=0.627-0.856)$ except at the reference site $(r=0.551)$. During $30 \% \mathrm{MVC}$, the thickness was significantly correlated with the CSA $(r=0.711-0.922)$ at all measurement sites. The correlation coefficients during $30 \% \mathrm{MVC}$ were significantly higher than those at rest.

\section{Discussion}

At all measurement sites, the thickness increased whereas the width decreased with $30 \% \mathrm{MVC}$ (Fig. 3). Correspondingly, the elbow flexor cross-sectional shapes changed greatly with $30 \% \mathrm{MVC}$. In the present condition, the gravitational force acts on the muscle shape in the anterior-posterior direction, suggesting that the cross-sectional shape of the relaxed muscle at rest sags in this direction owing to slackness. During contraction, however, the muscle slackness is removed. It is therefore likely that the stiffened muscle during contraction resists the gravitational force and thus its cross-sectional shape becomes thicker in the anterior-posterior direction compared with that at rest.

At levels from the reference site to $4 \mathrm{~cm}$ distal to it, the CSAs during $30 \% \mathrm{MVC}$ were significantly greater than those at rest (Fig. 3). As described in the earlier part, a contractioninduced decrease in muscle slackness should be one reason for this result. In addition, an increase in tendon length and a decrease in muscle length appear to affect the greater CSAs during 30\%MVC. When a joint is fixed at a given joint angle, a muscle-tendon unit is constant in length. However, a tendon is stretched during an isometric contraction (Ohta et al., 2005) and consequently muscle length is decreased. Given that muscle volume hardly changes by contraction (Baskin and Paolini, 1967), the aforementioned phenomenon can result in an increase in muscle CSA at certain sites.

On the other hand, the contraction-induced increase in the CSA was not found at the site $5 \mathrm{~cm}$ distal to the reference site (Fig. 3). As described above, muscle volume hardly changes by contraction (Baskin and Paolini, 1967). Hence, it is not surprising that the aforementioned contraction-induced increase in muscle CSA is not found at all measurement sites. In this study, due to the limitation of the MRI image quality, three muscles constituting elbow flexors (i.e., the biceps brachii, brachialis and brachioradialis) could not be clearly distinguished. They have different origins and insertions and thus the CSA distribution patterns along the upper arm length vary across the individual muscles (Kawakami et al., 1994). This fact suggests that the contraction-induced changes in the CSA distribution pattern are different among those muscles. The reasons for the discrepancy in the contraction-induced CSA changes among measurement sites should be investigated in more detail in future studies by employing advanced modality of tissue visualization that enables clear distinctions of the boundaries between muscles to be made.

The correlation coefficients between the thickness and CSA were significantly lower at rest than during 30\%MVC (Table 1). The thickness obtained here, defined as the vertical distance from the upper end of muscles (the elbow flexors) to that of bone (the humerus), is what the ultrasonography method defines as muscle thickness (Akagi et al., 2008, 2009, 2010; Candow and Chilibeck, 2005; Toji and Kaneko, 2007 etc.). Considering this fact, the above result brings up the question of the validity of using ultrasonic muscle thickness determined at rest to evaluate individual differences in muscle size. In previous studies, muscle thickness measurements with ultrasonography have been performed in standing (Abe et al., 1994; Akagi et al., 2010; Kubo et al., 2003a, 2003b; Miyatani et al., 2003, 2004; Pinkoski et al., 2006; Toji and Kaneko, 2007; Wakahara et al., 2010), supine (Ainscough-Potts et al., 2006; Arts et al., 2010; Campbell et al., 1995; Pillen et al., 2003, 2007; Reimers et al., 1998; Scholten et al., 2003), or prone (Candow and Chilibeck, 2005; Candow et al., 2006; Mohagheghi et al., 2007; Reimers et al., 1998) positions. In the standing position, the gravitational force acted on the muscles of interest in the proximal-distal direction. On the other hand, in the supine or prone position, the gravitational force acted in the anteroposterior direction of the examined muscles, ultrasonic muscle thickness values in which case would decrease or increase respectively. Thus, regardless of the subjects' posture, ultrasonic muscle thickness measurement at rest appears to include errors owing to the slackness of the relaxed muscles. For persons in certain pathological populations (e.g., complete spinal cord injury patients or stroke survivors) and/or elderly individuals, however, it is difficult to 
sustain muscle contractions. Furthermore, quantifying contraction intensity during the measurement of muscle thickness is not realistic in field studies examining larger samples. In these cases, it is difficult to apply the muscle thickness measurement under contracted condition to the thickness-based muscle size evaluation. In order to resolve these problems, therefore, it will be necessary to use other variables such as limb circumference (Akagi et al., 2008), limb length (Akagi et al., 2010; Miyatani et al., 2004), age (Akagi et al., 2010), and/or gender (Akagi et al., 2010), with the value of muscle thickness at rest, and to more accurately estimate the muscle CSA and/or volume as the previous studies performed.

In this study, the biceps brachii, brachialis, and brachioradialis were lumped together as the elbow flexors. Since the brachioradialis is located at the lateral side of the upper arm, the elbow flexor thickness measured at this position did not include the brachioradialis. In addition, the origin of the brachioradialis is at a more distal site than the reference site, and consequently, this muscle was not always included in the CSA at the present measurement sites. These facts could influence the current result on the thickness-CSA relationship of the elbow flexors. Nevertheless, the observed correlation coefficients between the thickness and the CSA were comparatively low at the more proximal sites where the brachioradialis was not included in the measurement of each of the two parameters. Hence, the existence of the brachioradialis should have only a minor effect on the present findings.

Before summarizing the results, limitations associated with the present approach should be addressed. In this study, the elbow flexors were examined because their thickness and CSA were expected to markedly increase with the low-intensity contraction. The observed phenomenon that thickness-based muscle size measurement can be affected by muscle slackness in the resting condition should not largely depend on the muscle fiber arrangements (i.e., parallel-fibered or pennate muscles), and thus we can say that the present findings are generalizable to other muscles. However, the extent of slackness of a muscle is suggested to be dependent on the angle of the joint that the muscle crosses (Herbert and Gandevia, 1995). This indicates that the contraction-induced changes in the elbow flexor cross-sectional shapes may be varied with the elbow joint angle, and consequently, the interpretation of the present results would be affected by the joint angle. This is a factor that needs to be addressed when the present finding is generalized to other muscles.

In summary, the elbow flexor cross-sectional shapes were thicker in the anterior-posterior direction during 30\%MVC than at rest. Additionally, significantly higher correlation coefficients were observed between muscle thickness and CSA during $30 \% \mathrm{MVC}$ than at rest. These results appear to be affected by difference in slackness in the elbow flexors between the conditions. The findings obtained here suggest that thickness-based size evaluation of a muscle includes the influence of muscle slackness on its cross-sectional shape when the thickness measurement is performed under relaxed conditions. Recognition of this methodological issue should be valuable for researchers who would like to evaluate muscle size using ultrasonography.

Acknowledgements We thank Dr. Tatsunori Matsui for his advice on the statistical analyses in the present study. This study was partly supported by a Grant-in-Aid for JSPS Fellows (No. 08J01634; R.A.) and a Grant-in-Aid for Young Scientists (Start-up) (No. 22800089; R.A.).

\section{References}

Abe T, Kondo M, Kawakami Y, Fukunaga T (1994) Prediction equations for body composition of Japanese adults by Bmode ultrasound. Am J Hum Biol 6: 161-170

Abe T, Kawakami Y, Suzuki Y, Gunji A, Fukunaga T (1997) Effects of 20 days bed rest on muscle morphology. J Gravit Physiol 4: S10-S14

Ainscough-Potts AM, Morrissey MC, Critchley D (2006) The response of the transverse abdominis and internal oblique muscles to different postures. Man Ther 11: 54-60

Akagi R, Kanehisa H, Kawakami Y, Fukunaga T (2008) Establishing a new index of muscle cross-sectional area and its relationship with isometric muscle strength. J Strength Cond Res 22: 82-87

Akagi R, Takai Y, Kato E, Fukuda M, Wakahara T, Ohta M, Kanehisa H, Kawakami Y, Fukunaga T (2009) Relationships between muscle strength and indices of muscle crosssectional area determined during maximal voluntary contraction in middle-aged and elderly individuals. J Strength Cond Res 23: 1258-1262

Akagi R, Takai Y, Kato E, Wakahara T, Ohta M, Kanehisa H, Fukunaga T, Kawakami Y (2010) Development of an equation to predict muscle volume of elbow flexors for men and women with a wide range of age. Eur J Appl Physiol 108: 689-694

Arts IM, Pillen S, Schelhaas HJ, Overeem S, Zwarts MJ (2010) Normal values for quantitative muscle ultrasonography in adults. Muscle Nerve 41: 32-41

Bamman MM, Newcomer BR, Larson-Meyer DE, Weinsier RL, Hunter GR (2000) Evaluation of the strength-size relationship in vivo using various muscle size indices. Med Sci Sports Exerc 32: 1307-1313

Baskin RJ, Paolini PJ (1967) Volume change and pressure development in muscle during contraction. Am J Physiol 213: $1025-1030$

Campbell IT, Watt T, Withers D, England R, Sukumar S, Keegan MA, Faragher B, Martin DF (1995) Muscle thickness, measured with ultrasound, may be an indicator of lean tissue wasting in multiple organ failure in the presence of edema. Am J Clin Nutr 62: 533-539

Candow DG, Chilibeck PD (2005) Differences in size, strength, and power of upper and lower body muscle groups in young and older men. J Gerontol A Biol Sci Med Sci 60: $148-156$ 
Candow DG, Chilibeck PD, Facci M, Abeysekara S, Zello GA (2006) Protein supplementation before and after resistance training in older men. Eur J Appl Physiol 97: 548-556

Gadeberg P, Andersen H, Jakobsen J (1999) Volume of ankle dorsiflexors and plantar flexors determined with stereological techniques. J Appl Physiol 86: 1670-1675

Hansen EA, Lee HD, Barrett K, Herzog W (2003) The shape of the force-elbow angle relationship for maximal voluntary contractions and sub-maximal electrically induced contractions in human elbow flexors. J Biomech 36: 17131718

Herbert RD, Gandevia SC (1995) Changes in pennation with joint angle and muscle torque: in vivo measurements in human brachialis muscle. J Physiol 484: 523-532

Hodges PW, Pengel LH, Herbert RD, Gandevia SC (2003) Measurement of muscle contraction with ultrasound imaging. Muscle Nerve 27: 682-692

Ikai M, Fukunaga T (1968) Calculation of muscle strength per unit cross-sectional area of human muscle by means of ultrasonic measurement. Int Z Angew Physiol 26: 26-32

Kawakami Y, Nakazawa K, Fujimoto T, Nozaki D, Miyashita M, Fukunaga T (1994) Specific tension of elbow flexor and extensor muscles based on magnetic resonance imaging. Eur J Appl Physiol Occup Physiol 68: 139-147

Kubo K, Kanehisa H, Azuma K, Ishizu M, Kuno SY, Okada M, Fukunaga T (2003a) Muscle architectural characteristics in women aged 20-79 years. Med Sci Sports Exerc 35: $39-44$

Kubo K, Kanehisa H, Azuma K, Ishizu M, Kuno SY, Okada M, Fukunaga T (2003b) Muscle architectural characteristics in young and elderly men and women. Int J Sports Med 24: 125-130

Leedham JS, Dowling JJ (1995) Force-length, torque-angle and EMG-joint angle relationships of the human in vivo biceps brachii. Eur J Appl Physiol Occup Physiol 70: 421-426

Miyatani M, Kanehisa H, Azuma K, Kuno S, Fukunaga T (2003) Site-related differences in muscle loss with aging "A cross-sectional survey on the muscle thickness in Japanese men aged 20 to 79 years." Int J Sport Health Sci 1: 34-40

Miyatani M, Kanehisa H, Ito M, Kawakami Y, Fukunaga T (2004) The accuracy of volume estimates using ultrasound muscle thickness measurements in different muscle groups. Eur J Appl Physiol 91: 264-272

Mohagheghi AA, Khan T, Meadows TH, Giannikas K, Baltzopoulos V, Maganaris CN (2007) Differences in gastrocnemius muscle architecture between the paretic and non-paretic legs in children with hemiplegic cerebral palsy. Clin Biomech (Bristol, Avon) 22: 718-724

Ohata K, Tsuboyama T, Haruta T, Ichihashi N, Kato T, Nakamura T (2008) Relation between muscle thickness, spasticity, and activity limitations in children and adolescents with cerebral palsy. Dev Med Child Neurol 50: $152-156$

Ohta M, Masuo Y, Kanehisa H, Kawakami Y, Fukunaga T (2005) Study on the application of the bio-electrical impedance method for the estimation of tendon elongation. Int J Sport Health Sci 3: 296-303

Pappas GP, Asakawa DS, Delp SL, Zajac FE, Drace JE (2002) Nonuniform shortening in the biceps brachii during elbow flexion. J Appl Physiol 92: 2381-2389

Pillen S, Scholten RR, Zwarts MJ, Verrips A (2003) Quantitative skeletal muscle ultrasonography in children with suspected neuromuscular disease. Muscle Nerve 27: 699-705

Pillen S, Verrips A, van Alfen N, Arts IM, Sie LT, Zwarts MJ (2007) Quantitative skeletal muscle ultrasound: Diagnostic value in childhood neuromuscular disease. Neuromuscul Disord 17: 509-516

Pinkoski C, Chilibeck PD, Candow DG, Esliger D, Ewaschuk JB, Facci M, Farthing JP, Zello GA (2006) The effects of conjugated linoleic acid supplementation during resistance training. Med Sci Sports Exerc 38: 339-348

Reimers CD, Harder T, Saxe H (1998) Age-related muscle atrophy does not affect all muscles and can partly be compensated by physical activity: An ultrasound study. J Neurol Sci 159: 60-66

Scholten RR, Pillen S, Verrips A, Zwarts MJ (2003) Quantitative ultrasonography of skeletal muscles in children: normal values. Muscle Nerve 27: 693-698

Sipila S, Suominen H (1991) Ultrasound imaging of the quadriceps muscle in elderly athletes and untrained men. Muscle Nerve 14: 527-533

Sipila S, Suominen H (1993) Muscle ultrasonography and computed tomography in elderly trained and untrained women. Muscle Nerve 16: 294-300

Toji H, Kaneko M (2007) Effects of aging on force, velocity, and power in the elbow flexors of males. J Physiol Anthropol 26: 587-592

Wakahara T, Takeshita K, Kato E, Miyatani M, Tanaka NI, Kanehisa H, Kawakami Y, Fukunaga T (2010) Variability of limb muscle size in young men. Am J Hum Biol 22: 55-59

Received: January 25, 2011

Accepted: April 18, 2011

Correspondence author: Ryota Akagi, Department of Sports Sciences, Japan Institute of Sports Sciences, 3-15-1 Nishigaoka, Kita-ku, Tokyo 115-0056, Japan

Phone: +81-3-5963-0231

Fax: +81-3-5963-0232

e-mail: carp_crespo_0522@ruri.waseda.jp 